\title{
CONSERVATION LAWS FOR SHALLOW WATER WAVES ON A SLOPING BEACH
}

\author{
YILMAZ AKYILDIZ \\ Department of Mathematical Sciences \\ University of Petroleum and Minerals \\ Dhahran, Saudi Arabia \\ (Received February 20, 1985)
}

\begin{abstract}
Shallow water waves are governed by a pair of non-linear partial differential equations. We transfer the associated homogeneous and non-homogeneous systems, (corresponding to constant and sloping depth, respectively), to the hodograph plane where we find all the non-simple wave solutions and construct infinitely many polynomial conservation laws. We also establish correspondence between conservation laws and hodograph solutions as well as Bäcklund transformations by using the linear nature of the problems on the hodogrpah plane.
\end{abstract}

KEY WORDS AND PHRASES. Water waves, conservation Zaws, Backlund transformation. 1980 AMS SUBJECT CLASSIFICATION CODE. $76 B 15$.

\section{INTRODUCTION.}

The linearity of a partial differential equation implies that any linear combination of solutions of the equation will also be a solution. This fundamental fact is also the main reason behind the method of separation of variables. In the event that a partial differential equation is non-linear, this property is lost, and it becomes impossible to employ separation of variable techniques, or any other argument that depends on superpossibility. Another striking difference between linear and non-linear partial differential equations is that, unlike linear p.d.e.'s, non-linear equations of ten do not admit solutions which can be continuously extended wherever the differential equations themselves remain regular.

During the last decade, finding exact solutions to non-linear differential equations has once more become important for both theoretical and practical purposes (Soliton Theory). It has been observed on some occasions (Korteweg-de Vries, SineGordon) that there are close connections between exact solutions, the existence of conservation laws, the inverse scattering method and Bäcklund transformations. Such cases are called completely integrable systems. They come in association with some linear differential equations. In this article we shall obtain similar relations and properties in the case of the shallow water wave theory.

We were introduced to the area of water waves by Nutku's recent paper [1]. Shallow water waves are governed by a system of two non-linear partial differential equations, 
which can also be written in the form of two conservation laws. First, we try to find further conservation laws by using the method of Estabrook-Wahlquist [2]. For the homogeneous case (corresponding to constant depth) we are able to construct an infinite family of conservation equations. This leads us to search for the exact solutions. It was at this point that we learnt that these results were already known to whitham [3]. We pass to the hodograph plane where we catch the linear system of equations associated with our non-linear problem. On this plane we show that conservation laws are easily derivable. On the hodograph plane we obtain all the solutions, except simple waves, by potentials which also satisfy linear equations. These potentials are, in fact, the Legendre transforms of the ones introduced by Nutku. Via these potentials we are also able to construct a correspondence between conservation laws and non-simple wave solutions of the homogeneous problem.

Finally, we take up the non-homogeneous case corresponding to a sloping beach. By using the polynomial conservation laws of the related homogeneous problem we construct an infinite family of polynomial conservation laws for the non-homogeneous case. By using the solutions of the cylindrical wave equation we also indicate how one can construct auto-Bäcklund and Bäcklund transformations for these homogeneous and nonhomogeneous problems.

2. METHOD OF ESTABROOK-WAHLQUIST.

We consider the following system of two homogeneous first order quasi-linear equations

$$
\begin{aligned}
& u_{t}+u u_{x}+2 c c_{x}=0, \\
& c_{t}+u_{x}+\frac{c}{2} u_{x}=0,
\end{aligned}
$$

representing shallow water waves, the bottom of the ocean being horizontal $[4] . u(x, t)$ and $c(x, t)$ are the velocities of the fluid and of the disturbance with respect to the fluid respectively. Subscripts denote partial derivatives.

First we shall apply the techniques of Estabrook-Wahlquist [2] (Section 3) to the system (2.1) and (2.2) above to find all the conservation laws, which are to be used to obtain potentials in their paper.

In the four-dimensional space of all the independent and dependent variables $\{x, t, u, c\}$, the set of first-order differential equations (2.1) and (2.2) above can be expressed by the following pair of differential 2-forms [1].

$$
\begin{aligned}
& \alpha_{1}=\mathrm{du} \wedge \mathrm{dx}-\mathrm{udu} \wedge \mathrm{dt}-2 \mathrm{cdc} \wedge \mathrm{dt}, \\
& \alpha_{2}=2 \mathrm{cdc} \wedge \mathrm{dx}-2 \mathrm{cudc} \wedge \mathrm{dt}-\mathrm{c}^{2} \mathrm{du} \wedge \mathrm{dt} .
\end{aligned}
$$

Any regular (differentiable) solution $(u, c)$ of (2.1) and (2.2) will annul this set of forms. Since $\mathrm{d} \alpha_{i}=0, i=1,2$, the ideal generated by $\alpha_{1}$ and $\alpha_{2}$ is closed and one can, therefore, apply Cartan's theory.

Conservation laws correspond to the existence of exact 2 -forms contained in the ring $\alpha_{i}$. Let us try to find all the 2-forms

$$
B=f \alpha_{1}+g \alpha_{2}
$$

satisfying $d B=0$, the condition for exactness. This is the (local) integrability 
condition for the existence of a 1 -form, say $\omega$, such that

$$
B=d \omega \text {. }
$$

The following treatment is restricted in that we do not allow $\mathrm{f}$ and $\mathrm{g}$ to be explicit functions of the independent variables $x$ and $t$. This seems plausible since the system (2.1) and (2.2) itself has no explicit $(x, t)$ dependence.

$$
\begin{aligned}
\mathrm{d} \beta & =\left(\mathrm{f}_{\mathrm{u}} \mathrm{du}+\mathrm{f}_{\mathrm{c}} \mathrm{dc}\right) \wedge \alpha_{1}+\left(\mathrm{g}_{\mathrm{u}} \mathrm{du}+\mathrm{g}_{\mathrm{c}} \mathrm{dc}\right) \wedge \alpha_{2} \\
& =\left(2 \mathrm{cg}_{\mathrm{u}}-\mathrm{f}_{\mathrm{c}}\right) \mathrm{du} \wedge \mathrm{dc} \wedge \mathrm{dx}+\left(\mathrm{c}^{2} \mathrm{~g}_{\mathrm{c}}-2 \operatorname{cug}_{\mathrm{u}}+\mathrm{uf}_{\mathrm{c}}-2 \mathrm{cf} \mathrm{f}_{\mathrm{u}}\right) \mathrm{du} \wedge \mathrm{dc} \wedge \mathrm{dt} .
\end{aligned}
$$

Hence, $d B=0$ implies

$$
\begin{aligned}
\mathrm{f}_{\mathrm{c}} & =2 \mathrm{cg}_{\mathrm{u}}, \\
2 \mathrm{f}_{\mathrm{u}} & =\mathrm{cg} \mathrm{g}_{\mathrm{c}} .
\end{aligned}
$$

$B=\mathrm{d} \omega=\mathrm{f} \alpha_{1}+\mathrm{g} \alpha_{2}=\mathrm{fdu} \wedge \mathrm{dx}+2 \mathrm{cgdc} \wedge \mathrm{dx}-\left(\mathrm{uf}+\mathrm{c}^{2} \mathrm{~g}\right) \mathrm{du} \wedge \mathrm{dt}-2 \mathrm{c}(\mathrm{f}+\mathrm{ug}) \mathrm{dc} \wedge \mathrm{dt}$, which, with the help of (2.7) and (2.8) integrates to

$$
\omega=d x \cdot \int f \partial u-d t \cdot \int\left(u f+c^{2} g\right) \partial u \text {. }
$$

Since $d \omega$ lies in a closed ideal of differential forms, "Frobenius theorem" applies: Any local solution which annuls the ideal must also annul $\omega$. This, in turn, gives us the following conservation equation.

$$
\mathrm{F}_{\mathrm{t}}+\mathrm{G}_{\mathrm{x}}=0
$$

where

$$
F=\int f \partial u \text { with } F_{c}=2 c g
$$

and

$$
G=\int\left(u f+c^{2} g\right) \partial u \text { with } G_{c}=2 c(f+u g) \text {. }
$$

When the condition (2.10) is satisfied we shall say that the pair (F,G) forms a consemation law. If $G=0$ at $x=0$ and $\infty$ we obtain the corresponding conserved quantity $\int_{0}^{\infty} \mathrm{Fdx}$.

Since the system of equations (2.1) and (2.2) is quasi-linear (i.e., linear in the derivatives) with polynomial coefficients in $u$ and $c$, the most interesting conservation equations are polynomial in $u$ and $c$. They may be obtained consistently from $(2.7)$ and (2.8) along with (2.11) and (2.12) by taking

$$
f=\sum_{i=0}^{n} p_{i}(u) c^{2 i}, g=\sum_{j=0}^{n} q_{j}(u) c^{2 j},
$$

from which it follows that

$$
\begin{aligned}
\mathrm{p}_{0}^{\prime} & =0, \\
\mathrm{q}_{\mathrm{n}}^{\prime} & =0, \\
\mathrm{mp}_{\mathrm{m}} & =\mathrm{q}_{\mathrm{m}-1}^{\prime}, \quad \mathrm{m}=1,2, \ldots \mathrm{n} \\
\mathrm{p}_{\mathrm{m}}^{\prime} & =\mathrm{mq}_{\mathrm{m}} .
\end{aligned}
$$

(It can easily be checked that the odd powers of $c$ do not survive). We list the first few of these polynomials: 


$\begin{array}{llll}\frac{f}{1} & \frac{g}{F} & \underline{G} \\ 0 & 0 & u & \frac{u^{2}}{2}+z^{2} \\ c^{2} & 1 & c^{2} & u c^{2} \\ u c^{2} & u & u c^{2} & u^{2} c^{2}+\frac{c^{4}}{2} \\ u^{2} c^{2}+c^{4} & \frac{u^{2}}{2}+c^{2} & \frac{u^{2} c^{2}}{2}+\frac{c^{4}}{2} & \frac{u^{3} c^{2}}{2}+u c^{4} \\ \frac{2}{3} u^{3} c^{2}+2 u c^{4} & \frac{u^{3}}{3}+2 u c^{2} & \frac{u^{3} c^{2}}{3}+u c^{4} & \frac{u^{4} c^{2}}{3}+\frac{3 u^{2} c^{4}}{2}+\frac{c^{6}}{3} \\ & \frac{u^{4}}{6}+2 u^{2} c^{2}+c^{4} & \frac{u^{4} c^{2}}{6}+u^{2} c^{4}+\frac{c^{6}}{3} & \frac{u^{5} c^{2}}{6}+\frac{4}{3} u^{3} c^{4}+u c^{6}\end{array}$

It is interesting to note that $F$ and $G$ are homogeneous in $u$ and $c$. This observation immediately makes us think of our Russian colleagues who have extracted the algebro-geometric structures of some of the "completely integrable" evolution equations $[5,6]$. For the boundary conditions $u=0, c=0$ at $x=0$ and $\infty$ we obtain infinitely many conserved quantities by integrating $F^{\prime}$ 's with respect to $x$ from 0 to $\infty$.

Differentiating (2.7) partially with respect to $u$, and (2.8) with respect to $c$ and subtracting, we find

$$
4 g_{\text {uu }}=g_{c c}+\frac{g_{c}}{c}
$$

or

$$
4 g_{\mathrm{uu}}=\frac{1}{\mathrm{c}}\left(\mathrm{cg}_{\mathrm{c}}\right)_{\mathrm{c}}
$$

Thus, we have the cylindrical wave equation for $g(u, c)$. This is a linear equation for $g$ which can be solved by standard methods. Similarly, for $f(u, c)$ we have

$$
4 \mathrm{f}_{\mathrm{uu}}=\mathrm{f}_{\mathrm{cc}}-\frac{\mathrm{f}}{\mathrm{c}} \text {, }
$$

or

$$
4 \mathrm{f}_{\mathrm{uu}}=\mathrm{c}\left(\frac{\mathrm{f}}{\mathrm{c}}\right)_{\mathrm{c}} .
$$

On the other hand, upon eliminating $f$ and $g$ from the set of equations (2.11) and (2.12) we arrive at the following relations:

$$
\begin{aligned}
& G_{u}=u F_{u}+\frac{c_{1}}{2} F_{c}, \\
& G_{c}=2 c F_{u}+u F_{c} .
\end{aligned}
$$

As before, differentiating (2.16) partially with respect to $c$, and differentiating (2.17) partially with respect to $u$ and subtracting we obtain

$$
4 \mathrm{~F}_{\mathrm{uu}}=\mathrm{F}_{\mathrm{cc}}-\frac{\mathrm{F}_{\mathrm{c}}}{\mathrm{c}} \text {. }
$$

Unfortunately, (maybe fortunately), we don't have a nice equation for $G$. We make the following observation: Even though $x$ and $t$ are the independent variables, all our expressions are (linear) partial differential equations in the 
variables $u$ and $c$. This is because we have no $(x, t)$ dependence in the system of equations (2.1) and (2.2) with which we started. This suggests that we should interchange the roles of the dependent and independent variables. This is called the "hodograph" method, which we will take up in the following section.

3. METHOD OF HODOGRAPH TRANSFORMATION.

We consider the system (2.1) and (2.2) which has no explicit $(x, t)$ dependence. For any region where the Jacobian

$$
J=u_{x} c_{t}-u_{t} c_{x}
$$

is non-zero, the system (2.1) and (2.2) can be transformed into an equivalent linear system by interchanging the roles of dependent and indepe dent vari blei. $f J \neq 1$ for a solution $u(x, t), c(x, t)$ of $(2.1)$ and $(2.2)$, we may consider $x$ and $t$ as functions of $u$ and $c$. From

$$
\begin{aligned}
& \mathrm{u}_{\mathrm{x}}=\mathrm{J} \mathrm{t}_{\mathrm{c}}, \mathrm{u}_{\mathrm{t}}=-\mathrm{J \textrm {x } _ { \mathrm { c } }}, \\
& \mathrm{c}_{\mathrm{x}}=-\mathrm{J} \mathrm{t}_{\mathrm{u}}, \mathrm{c}_{\mathrm{t}}=\mathrm{Jx_{ \textrm {u } }}
\end{aligned}
$$

we see that the highly non-linear factor $J$ cancels through in (2.1) and (2.2) and that $x(u, c)$ and $t(u, c)$ satisfy the linear differential equations

$$
\begin{aligned}
& x_{u}=u t_{u}-\frac{c}{2} t_{c}, \\
& x_{c}=-2 c t_{u}+u t_{c} .
\end{aligned}
$$

By elimimating $x$ we obtain the linear equation

$$
4 t_{u u}=t_{c c}+\frac{3}{c} t_{c} \text {, }
$$

which can be solved by standard methods. This can be further simplified by introducing the transformation

$$
t=\frac{s_{c}}{c}
$$

We obtain the cylindrical wave equation

$$
4 s_{u u}=s_{c c}+\frac{s_{c}}{c}
$$

whose solutions involve Bessel functions.

We remark that $g$ and $s$ satisfy the same equation.

The described transformation of the $(x, t)-p l a n e$ into the $(u, c)-p l a n e$ is called a hodograph transformation. Since the possibility of this reduction dependes essentially on the assumption $J \neq 0$, solutions for which $J=0$ cannot be obtained by the hodograph method. These solutions are called simple waves and they are important tools for the solutions of flow problems, (Courant-Friedrichs [7], Section 29). Wave-breaking occurs when $J=0$ corresponding to the multivaluedness, i.e., shock waves. We notice that the solution

$$
u=\frac{2}{3} \frac{x}{t}, c=\frac{1}{3} \frac{x}{t},
$$

given by the Nutku [1] represents a simple wave. So, we could not possibly obtain this solution by the hodograph method.

We would like to mention that in the set of all solutions the waves form a set of measure zero. But this is not to say simple waves are unimportant. 
Just to show how noatural it is to work in the hodograph plane we shall rederive the conservation equations $(2.16)$ and $(2.17)$. In the $(u, c)-p l a n e$ the equation $(2.10)$ becomes

$$
\begin{aligned}
F_{t}+G_{x} & =F_{u_{t} u_{t}}+F_{c} c_{t}+G_{u} u_{x}+G_{c} c_{x} \\
& =-J x_{c} F_{u}+J x_{u} F_{c}+J t_{c} G_{u}-J t_{u} G_{c}=0
\end{aligned}
$$

Above we have employed the equations (3.1) and (3.2). Again, the non-linear factor J cancels through and we arrive at

$$
x_{u} F_{c}-x_{c} F_{u}=t_{u} G_{c}-t_{c} G_{u}
$$

or

$$
\mathrm{dx} \wedge \mathrm{dF}=\mathrm{dt} \wedge \mathrm{dG} \text {. }
$$

Upon using (3.3) and (3.4), (3.8) becomes

$$
t_{c}\left(G_{u}-u F u-\frac{c}{2} F_{c}\right)=t_{u}\left(G_{c}-2 c F_{u}-u F_{c}\right)
$$

Since this is to be an identity, the coefficients of the derivatives must vanish separately:

$$
\begin{aligned}
& G_{u}=u F_{u}+\frac{c}{2} F_{c}, \\
& G_{c}=2 c F_{u}+u F_{c} .
\end{aligned}
$$

These are the same as $(2.16)$ and $(2.17)$. We note that the computation above is somewhat shorter than the Estabrook-Wahlquist method used in the previous section to establish these equations. Whitham [3] has an even simpler way of deriving them. Even so, we have included the method of Estabrook-Wahlquist because it has provided us with two nice functions - $\mathrm{f}$ and $\mathrm{g}$ - of which we make use in this paper.

4. POTENTIALS.

We look for potentials in the hodograph plane. The system of equations (3.3) and (3.4) can be rewritten in the following equivalent form

$$
\begin{gathered}
(2 c x-2 c u t)_{u}=-\left(c^{2} t_{c}\right), \\
(x-u t)_{c}=-(2 c t)_{u} .
\end{gathered}
$$

These, in return, suggest the existence of potentials $\Psi(u, c)$ and $\Phi(u, c)$ satisfying

$$
\Psi_{u}=-c^{2} t, \quad \psi_{c}=2 c x-2 c u t,
$$

and

$$
\Phi_{u}=x-u t, \Phi_{c}=-2 c t \text {. }
$$

$\Psi, \Phi$ are in fact, the Legendre transforms of the potentials introduced by Nutku [1]. Solving them for $x$ and $t$ we obtain

$$
\mathrm{x}=\frac{\Psi_{\mathrm{c}}}{2 \mathrm{c}}-\frac{\mathrm{u}}{\mathrm{c}^{2}} \psi \mathrm{u}, \mathrm{t}=-\frac{\psi_{\mathrm{u}}}{\mathrm{c}^{2}}
$$

and

$$
\mathrm{x}=\Phi_{\mathrm{u}}-\frac{\mathrm{u}}{2 \mathrm{c}} \Phi_{\mathrm{c}}, \mathrm{t}=-\frac{\Phi_{c}}{2 \mathrm{c}}
$$

Hence, if we know $\Psi(u, c)$ or $\Phi(u, c)$, by using these formulas we con compute $x$ and t. Combining $(4.5)$ and $(4.2)$ we obtain

$$
4 \psi_{\mathrm{uu}}=\psi_{\mathrm{cc}}-\frac{\Psi_{\mathrm{c}}}{\mathrm{c}} .
$$


(4.6) together with (4.7) gives

$$
4 \phi_{\mathrm{uu}}+\Phi_{\mathrm{cc}}+\frac{\Phi_{\mathrm{c}}}{\mathrm{c}}
$$

Unlike their legendre transforms, $\Psi$ and $\Phi$ satisfy linear equations.

Comparing (2.13), (2.15), (2.18), (3.7), (4.7), and (4.8), our readers realize that we keep encountering the following set of equations:

$$
4 x_{\mathrm{uu}}=x_{\mathrm{cc}} \pm \frac{x_{\mathrm{c}}}{\mathrm{c}} \text {. }
$$

In the next section too we shall encounter these equations when we are dealing with a related non-homogeneous problem. Not only can we derive the conservation laws from the solutions of (4.9) but we can also construct all the hodograph solutions of the original system of equations with which we started. In this way, we are also able to construct a solution of the system of equations (2.1) and (2.2) from a given conservation law by letting $\psi=F$ and by using (4.5). We can reverse this process for nonsimple wave solutions. Now we have an infinite family of solutions associated with the list of polynomial conservation laws given in Section 2 . Here we list the first few of these special solutions:

$\begin{array}{lllll}\underline{F} & \underline{x} & \frac{t}{u} & \underline{u} & \frac{c^{2}}{c^{2}} \\ u & 1 & \frac{1}{c^{2}} & \frac{x}{t} & \frac{1}{t} \\ c^{2} & 0 & 0 & - & - \\ u c^{2} & 1 & - & x+\frac{t^{2}}{2} \\ \frac{u^{2} c^{2}}{2}+\frac{c^{4}}{2} & -\frac{u^{2}}{2}+c^{2} & -u & -t & \text { implicit solution } \\ \frac{u^{3} c^{2}}{3}+u c^{4} & \frac{2 u^{3}}{3}-u c^{2} & u^{2}+c^{2} & \text { implicit solution } \\ \frac{u^{4} c^{2}}{6}+u^{2} c^{4}+\frac{c^{6}}{3} & \frac{u^{4}}{2}-c^{4} & \frac{2 u^{3}}{3}+2 u c^{2} & \end{array}$

5. CASE OF SLOPING BEACH.

We consider the following non-homogeneous system of equations

$$
\begin{aligned}
& u_{t}+u u_{x}+2 c c_{x}=g \beta, \\
& c_{t}+u c_{x}+\frac{c}{2} u_{x}=0,
\end{aligned}
$$

representing shallow water waves on a sloping beach. The constant term $g B$ involves the gravitational constant $g$ and the slope of the bottom $B$.

On p. 70 of his Tata Institute Notes [8], Whitham absorbs the non-homogeneous term $g \beta$ in a conservation form as

$$
(u-g \beta t)_{t}+\left(\frac{1}{2} u^{2}+c^{2}\right)_{x}=0,
$$

and adds the following statement: "But this comment does not appear to lead any further." However, by the means of (5.3) we were fortunate in finding ourselves able $t$. construct conservation laws in the form

$$
\left[F(u, c)-\sum_{i=1}^{m} \frac{1}{i !}(g \beta t){ }^{i} P_{i}(u, c)\right]_{t}+\left[G(u, c)-\sum_{i=1}^{m} \frac{1}{i !}(g \beta t){ }^{i} Q_{i}(u, c)\right]_{x}=0,
$$


for the non-homogeneous system (5.1) and (5.2) above. We will denote the contents of the two square brackets in $(5.4)$ as $\tilde{\mathrm{F}}$ and $\tilde{\mathrm{G}}$ respectively. As one can guess, we shall require $(F, G)$ to form a conservation law for the related homogeneous system (2.1) and (2.2). Hence, as in (2.16) and (2.17) of Section 2 they satisfy the following linear system of equations

$$
\begin{aligned}
& G_{u}=u F_{u}+\frac{c}{2} F_{c}, \\
& G_{c}=2 c F_{u}+u F_{c},
\end{aligned}
$$

whose integrability condition is (2.18)

$$
4 \mathrm{~F}_{\mathrm{uu}}=\mathrm{F}_{\mathrm{cc}}-\frac{\mathrm{F}_{\mathrm{c}}}{\mathrm{c}} \text {. }
$$

With the help of $(5.5)$ and $(5.6),(5.4)$ simplifies to

$$
\begin{aligned}
& g \beta \cdot F_{u}-\sum_{i=1}^{m} \frac{1}{i !}(g \beta t)^{i}\left\{g \beta \cdot P_{i u}+u_{x}\left(Q_{i u}-u P_{i u}-\frac{c}{2} P_{i c}\right)+\right. \\
& \left.c_{x}\left(Q_{i c}-2 c P_{i u}-u P_{i c}\right)\right\}-g \beta \cdot \sum_{i=1}^{m} \frac{1}{(i-1) !}(g \beta t){ }^{i-1} P_{i}=0 .
\end{aligned}
$$

Imposing the following further conditions

$$
\begin{aligned}
& Q_{i u}=u P_{i u}+\frac{c}{2} P_{i c}, \\
& Q_{i c}=2 c P_{i u}+u P_{i c},
\end{aligned}
$$

forces us to take

$$
\begin{aligned}
& \mathrm{P}_{1}=\mathrm{F}_{\mathrm{u}} \text {, } \\
& \mathrm{P}_{2}=-\mathrm{P}_{\mathrm{lu}}=-\mathrm{F}_{\mathrm{uu}} \text {, } \\
& \mathrm{P}_{3}=-\mathrm{P}_{2 \mathrm{u}}=+\mathrm{F}_{\text {uuu }} \text {, } \\
& \dot{\mathrm{P}}_{\mathrm{m}}=-\mathrm{P}_{\mathrm{m}-1, \mathrm{u}}=(-1)^{\mathrm{m}+1} \mathrm{~F}_{\mathrm{uu}, . . \mathrm{u}} \text {, } \\
& \text { m-times }
\end{aligned}
$$

and

$$
\mathrm{P}_{\mathrm{mu}}=0 .
$$

For convenience, we shall use the notation $\mathrm{F}_{\mathrm{u}}^{(\mathrm{m})}=\underset{\mathrm{m}-\mathrm{times}}{\mathrm{F}_{\text {uu...u. }}}$ The last condition requires us to take $\mathrm{F}_{\mathrm{u}}^{(\mathrm{m}+1)}=0$, which can automatically be satisfied for a suitable $F$, if we start with polynomial conservation laws for the related homogeneous problem. What really makes this construction work is the fact that all the $P_{i}$ turn out to be $\pm F_{u}^{(i)}$ and the pairs $\left(P_{i}, Q_{i}\right)$ and $(F, G)$ satisfy the same system of equations. Since the compatibility equation $(5.7)$ is also satisfied by the u-derivatives $F_{u}^{(i)}$, The integrability condition of the system (5.8) and (5.9) is automatically guaranteed. We have, therefore, a consistent method, and by using the list on p. in Section 2, we can construct an infinite family of conservation laws for the non-homogeneous system (5.1) and (5.2). 
The computations for $Q_{i}$ become easier once one realizes that the $i$ th u-derivative of $F$ on the $j$ th line in the list is proportional to $F$ on the $(j-i)$ th line in the same 1 ist in Section 2 (excluding the first line).

Here we list the first few of these conservation laws $(\tilde{F}, \tilde{C})$ :

$$
\begin{aligned}
& \tilde{\tilde{F}} \quad \tilde{C} \\
& u-g P t \quad \frac{u^{2}}{2}+c^{2} \\
& \mathrm{c}^{2} \quad \mathrm{uc}^{2} \\
& u c^{2}-g \beta t c^{2} \quad u^{2} c^{2}+\frac{c^{4}}{2}-g \beta t u c^{2} \\
& \frac{u^{2} c^{2}}{2}+\frac{c^{4}}{2}-g B t u c^{2}+\frac{1}{2}(g B t)^{2} c^{2} \quad \frac{u^{3} c^{2}}{2}+u c^{4}-g B t\left(u^{2} c^{2}+\frac{c^{4}}{2}-\right)+\frac{1}{2}(g B t)^{2} u c^{2} \\
& \frac{u^{3} c^{2}}{3}+u c^{4}-2 g B t\left(\frac{u^{2} c^{2}}{2}+\frac{c^{4}}{2}\right) \quad \frac{u^{4} c^{2}}{3}+\frac{3 u^{2} c^{4}}{2}+\frac{c^{6}}{3}-2 g B t\left(\frac{u^{3} c^{2}}{2}+u c^{4}\right) \\
& +(g \beta t)^{2} u c^{2}-\frac{1}{3}(g \beta t)^{3} c^{2}+(g \beta t)^{2}\left(u^{2} c^{2}+\frac{c^{4}}{2}\right)-\frac{1}{3}(g \beta t)^{3} u c^{2} \\
& \frac{u^{4} c^{2}}{6}+u^{2} c^{4}+\frac{c^{6}}{3}-2 g \beta t\left(\frac{u^{3} c^{2}}{3}+u c^{4}\right) \quad \frac{u^{5} c^{2}}{6}+\frac{4 u^{3} c^{4}}{3}+u c^{6}-2 g \beta t\left(\frac{u^{4} c^{2}}{3}+\frac{3 u^{2} c^{4}}{2}+\frac{c^{6}}{3}\right) \\
& +2(g B t)^{2}\left(\frac{u^{2} c^{2}}{2}+\frac{c^{4}}{2}\right) \quad+2(g B t)^{2}\left(\frac{u^{3} c^{2}}{2}+u c^{4}\right) \\
& -\frac{2}{3}(g \beta t)^{3} u c^{2}+\frac{1}{6}(g \beta t)^{4} c^{2} \quad-\frac{2}{3}(g \beta t)^{3}\left(u^{2} c^{2}+\frac{c^{4}}{2}\right)+\frac{1}{6}\left(g \beta t ; u^{4} u c^{2}\right.
\end{aligned}
$$

We note that $\tilde{F}$ and $\tilde{G}$ are homogeneous in $u, c$, and $t$. We would like to thank Dr. Mirie for drawing our attention to the fact that the terms containing $t$ can be put into the form $(u-g \beta t)^{i}$. But, as is clear even from the first line of the above list, we cannot completely eliminate all the $u^{\prime} s$ in $\tilde{G}^{\prime} s$, although we can write $\tilde{F}^{\prime} s$ in terms of $\mathrm{v}=\mathrm{u}-\mathrm{g} \beta \mathrm{t}$ and $\mathrm{c}$ only. Nevertheless, $v^{\prime}$ 's do not show up separately in $\tilde{G}^{\prime} s$; they all come multiplied with u's or c's. Hence for the boundary conditions $u=0, c=0$ at $x=0$ and $\infty$ we still obtain infinitely many conserved quantities by integrating $\tilde{F}^{\prime} s$ with respect to $x$ from 0 to $\infty$, cf. the homogeneous case.

Having constructed an infinite number of conservation laws, one might, therefore, expect to be able to find the solution of the non-homogeneous system (5.1) and (5.2) analytically. Indeed, as we have learnt from Whitham [8], Carrier and Greenspan introduced new variables suggested by the characteristic forms of these equations and applied a hodograph transformation to them and obtained

$$
\begin{aligned}
& \mathrm{g} \beta \mathrm{x}=-\frac{\phi_{\lambda}}{2}+\frac{\phi_{\sigma}^{2}}{2 \sigma^{2}}+\frac{\sigma^{2}}{16}, \\
& \mathrm{~g} B \mathrm{t}=\frac{\lambda}{4}-\frac{\phi_{\sigma}}{\sigma},
\end{aligned}
$$

where $\sigma=4 c, \lambda=-4(u-g \beta t)$ and $\phi$ satisfies the cylindrical wave equation

$$
4 \phi_{\lambda \lambda}=\phi_{\sigma \sigma}+\frac{\phi_{\sigma}}{\sigma} \text {. }
$$


We observe that $\phi$ in (5.12) and $\phi$ in (4.8) satisfy the same kind of equation. Hence, after the necessary relabelling of the variables, a solution of (5.12) can be used to generate a solution of either of the problems: homogenequs (via (5.11) and non-homogeneous (via (5.10) and (5.11)). In this way, we find a correspondence between the non-simple wave solutions of the two systems which we have considered in this paper. In a way, this correspondence can be thought of as a Bäcklund transformation between the homogeneous and non-homogeneous problems (2.1) and (2.2), and (5.1) and (5.2). By using the 1inearity of the space of solutions of (5.12) we can also construct autoBäcklund transformations for each of these problems.

We leave it to our interested readers to construct the solutions of the nonhomogeneous problem which correspond to the solutions given in Section 4 of the homogeneous system.

To us, the story of this paper looks similar to the Hydrogen Atom problem, (the invariance group being the space of solutions of the cylindrical wave equation). We expect to shed more light on this subject by using the orbit theory picture of KrillovKostant-Souriau. This is our forthcoming project.

ACKNOWLEDGEMENTS. I would like to apologize Ayshe/Ruth for the sleepless Arabian Nights and to express my appreciation of the UPM-Beach where I meet the real waves and enjoy them. Also, special thanks to R.O. Wells, Jr. and G. Bluman for their encouraging comments.

\section{REFERENCES}

1. NUTKU, Y. "Potentials for Non-linear Shallow Water Waves", Preprint(1981).

2. WAHLQUIST, H.D. and ESTABROOK, F.B. "Prolongation Structure of Non-1inear Evolution Equations", J. Math. Phys. 16 1, (1975).

3. WhithAM, G.B. "Linear and Non-1inear Waves", John Wiley \& Sons, New York, 1974.

4. STOKER, J.J. "Water Waves", Interscience Publishers, New York, 1975.

5. DRINFELD, V.G., KRICHEVER, I.M., MANIN, J.I., and NOVIKOV, S.P. "Methods of Algebraic Geometry in Contemporary Mathematical Physics", Soviet Science Reviews, Phys. Reviews, 1978, Over. Pub. Ass., Amsterdam 1980 .

6. KRICHEVER, I.M. "The Method of Algebraic Geometry in the Theory of Non-linear Equations", Us pekhi. Math. Nauk 32, 183-208, (1977).

7. COURANT, R., and FRIEDRICHS, K.0. "Supersonic Flow and Shock Waves", SpringerVerlag, New York, 1972 .

8. WhithaM, G.B. "Lectures on Wave Propagation", Tata Institute, Bombay, 1979. 


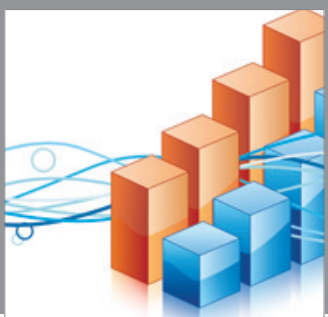

Advances in

Operations Research

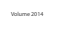

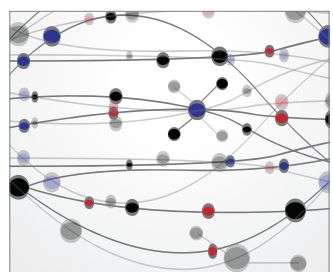

\section{The Scientific} World Journal
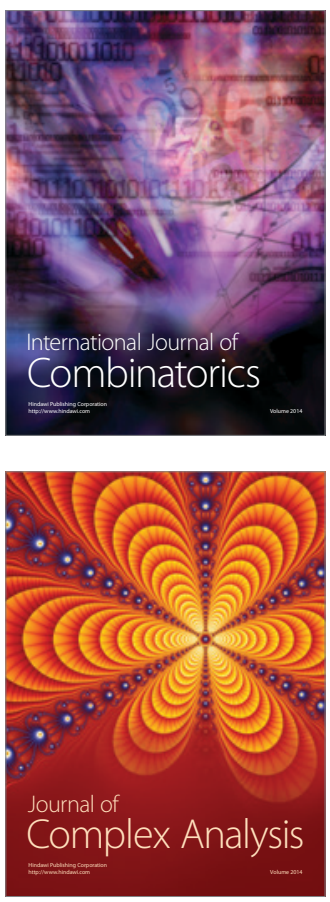

International Journal of

Mathematics and

Mathematical

Sciences
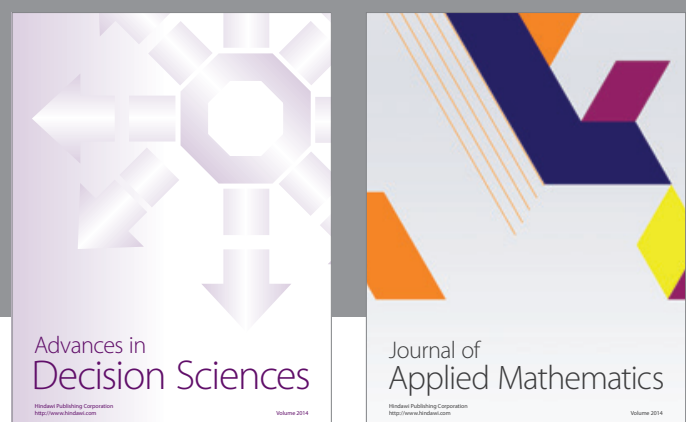

Journal of

Applied Mathematics
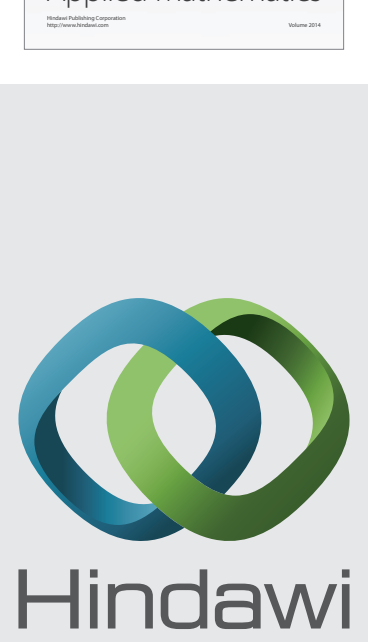

Submit your manuscripts at http://www.hindawi.com
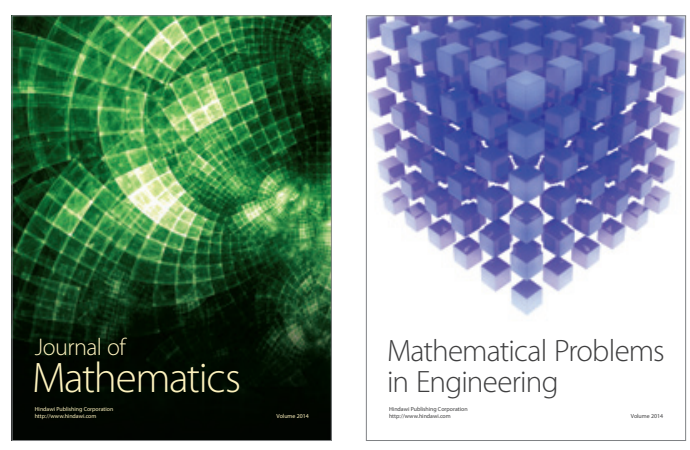

Mathematical Problems in Engineering
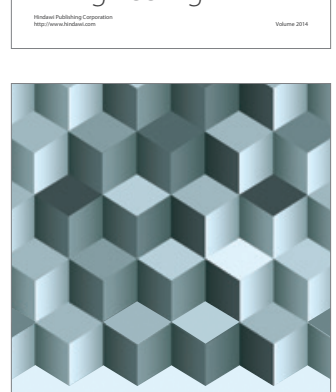

Journal of

Function Spaces
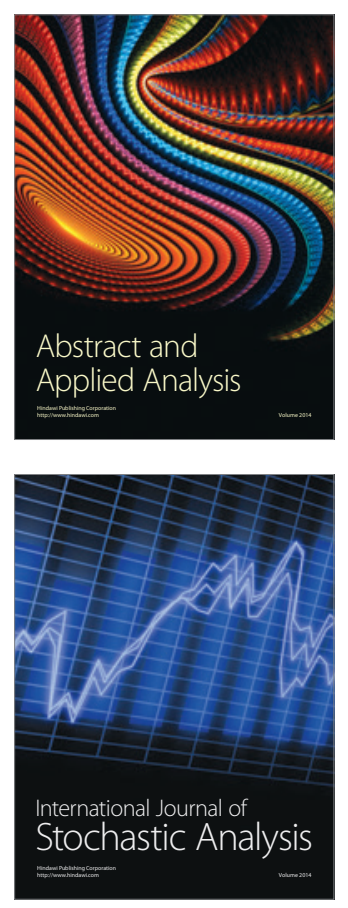

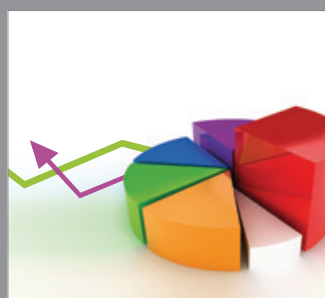

ournal of

Probability and Statistics

Promensencen
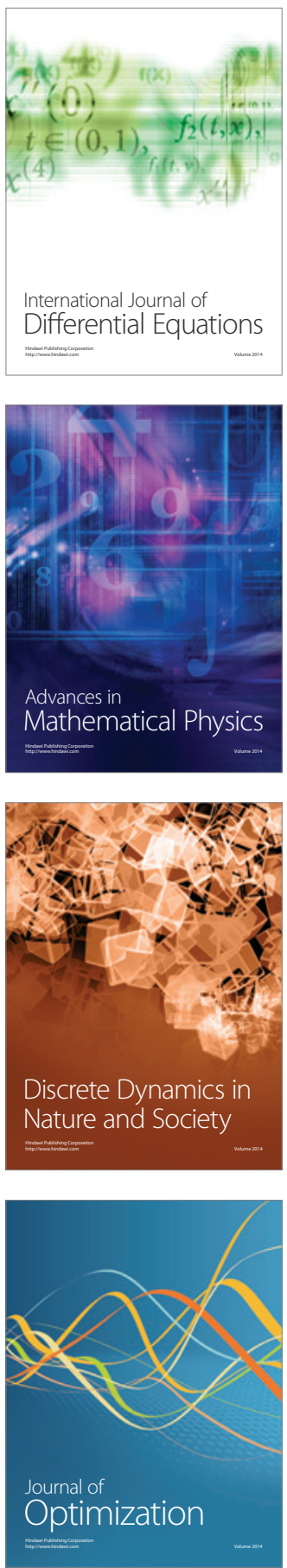